\title{
Abate halal com e sem insensibilização em ovinos: implicações sobre o bem-estar animal e a eficiência da sangria
}

[Halal slaughter with and without stunning in sheep: implications on animal welfare and bleeding efficiency]

\author{
A. Sterza ${ }^{1}$, M.K. Falbo ${ }^{2 *}$, I.E. Sandini ${ }^{2}$, D.D. Pivatto ${ }^{3}$ \\ ${ }^{1}$ Aluna de pós-graduação - Universidade Estadual do Centro Oeste - Guarapuava, PR \\ ${ }^{2}$ Universidade Estadual do Centro Oeste - Guarapuava, PR \\ ${ }^{3}$ Médica veterinária autônoma - Guarapuava, PR
}

\begin{abstract}
RESUMO
O objetivo deste estudo foi avaliar as implicações sobre o bem-estar animal e a eficiência da sangria no abate halal com e sem insensibilização em ovinos. Foram avaliados 102 ovinos, em abate comercial, separados randomicamente pelo peso em dois grupos: com insensibilização (CI) e sem insensibilização (SI). Verificou-se que os animais sem insensibilização apresentaram sinais de endireitamento e vocalização após 20 segundos da degola. Houve diferença significativa para hemácias $(\mathrm{P}<0,01)$, hemoglobina $(\mathrm{P}<0,01)$, leucócitos totais $(\mathrm{P}<0,001)$, bem como para os biomarcadores de estresse, como $\mathrm{CK}(\mathrm{P}<0,01)$ e lactato $(\mathrm{P}<0,001)$. Apesar de não apresentarem diferença estatística significativa, o LDH e o cortisol ficaram acima dos valores basais em ambos os grupos. Não foram observadas alterações séricas para glicose e AST. Houve diferença estatística significativa para eficiência da sangria $(\mathrm{P}<0,01)$. Os resultados demonstraram que houve estresse no abate halal com e sem insensibilização e melhor eficiência da sangria em animais não insensibilizados.
\end{abstract}

Palavras-chave: cortisol, atordoamento, exsanguinação, abate muçulmano

\begin{abstract}
The objective of this study was to evaluate the implications on animal welfare and the sangria efficiency in halal slaugther with and without stunning in sheep. 102 sheep were appraised in a commercial slaugthter, divided randomly for the weight in two groups: with stunning (CI) and without stunning (SI). It was verified that the animals without stunning presented straighten signs and vocalization after 20 seconds of decapitation. There was significant difference for erythrocytes $(P<0.05)$, hemoglobin $(P<0.01)$, leucocytes $(P<0.01)$, stress of biomarkers $C K(P<0.01)$ and lactate $(P<0.01)$. Alterations in serum were not observed for glucose and AST. There was significant statistical difference for bleeding efficiency $(P<0.01)$. The results demonstrated that there was stress in the halal slaugther with and without stunning and better bleeding efficiency in animals with traditional religious slaughter without stunning.
\end{abstract}

Keywords: cortisol, stunning, exsanguination, Muslim slaughter

\section{INTRODUÇÃO}

Estima-se que, até 2020, um terço da população mundial seja muçulmana. E, com esse cenário, estima-se um crescimento de $62,3 \%$ do mercado muçulmano para o comercio de carne halal (Relatório..., 2017). O abate halal tradicional é realizado sem o atordoamento do animal antes da degola e, nos países mais tradicionais, como Irã e
Arábia Saudita, os muçulmanos destacam sua preferência pelo abate sem insensibilização, por acreditarem que a exsanguinação seja mais eficiente (Fuseini et al., 2017), porém, do ponto de vista do bem-estar animal, esse abate ainda é bastante controverso (Grandin, 2017). Uma das preocupações se refere ao tempo em que o animal perde a consciência e ao tempo em que ele pode sentir dor após a degola (Gregory et al., 2010).

Recebido em 24 de maio de 2019

Aceito em 5 de maio de 2020

*Autor para correspondência (corresponding author)

E-mail: margaretefalbo@hotmail.com 
Embora a ausência de insensibilização seja permitida no Brasil em abates religiosos (Brasil..., 2000), vários países muçulmanos aceitam alguns métodos de insensibilização, desde que o animal permaneça vivo no momento da degola (Fuseini et al., 2016). Em ovinos é realizada a insensibilização elétrica apenas na cabeça do animal, por três segundos (World..., 2019). Porém, Farouk et al. (2014) relataram incidências de hemorragias, hematomas, petéquias, equimoses e fraturas ósseas em cordeiros atordoados usando apenas choque elétrico na cabeça, interferindo na "qualidade espiritual" da carne. Assim, este trabalho teve como objetivo avaliar o abate halal comercial em ovinos com e sem insensibilização, bem como suas implicações sobre o bem-estar animal e a eficiência da sangria.

\section{MATERIAL E MÉTODOS}

Esta pesquisa foi aprovada pelo Comitê de Ética no Uso de Animais-Ceua, sob protocolo $\mathrm{n}^{\circ}$ 037/2017, da Universidade Estadual do CentroOeste. Foi acompanhado o abate comercial de 102 ovinos (45 a 70kg), mestiços Ile de France $\mathrm{x}$ Corriedale, em um frigorífico inspecionado pelo Serviço de Inspeção Estadual do Paraná (SIE), no período de quatro semanas consecutivas. Todos os animais chegavam ao abatedouro pelo menos 12 horas antes do abate. Cada grupo foi mantido em alojamento separado e passou por descanso, jejum e dieta hídrica, conforme as normas de bem-estar animal (Brasil..., 2000).

Para cada lote de animais abatidos semanalmente, dividia-se o número total de animais, separados randomicamente pelo peso, em dois grupos. $\mathrm{O}$ grupo sem insensibilização (SI) obedecia ao seguinte protocolo: os animais entravam no local de insensibilização e dois funcionários faziam a contenção do animal em decúbito lateral sobre uma mesa com grade metálica; um dos funcionários segurava as patas dianteiras e traseiras do animal, e o outro estendia o pescoço do animal para o Zabeh (muçulmano autorizado a realizar o abate halal) realizar a degola. Neste grupo, foi avaliada a sensibilidade dos animais 20 segundos após a sangria. Durante a sangria, o animal permanecia em decúbito lateral por três minutos.

No outro grupo, com insensibilização (CI), os animais entravam no local de insensibilização e dois funcionários faziam a contenção, para colocação dos eletrodos atrás da orelha, com um aparelho no formato de tesoura (Dalpino INS $01^{\circledR}$ Santo André/SP), amperagem do choque de 0,5 a 2 amperes e voltímetro regulável para $350 \mathrm{~V}$ a $750 \mathrm{~V}$ (trezentos e cinquenta a setecentos e cinquenta volts), variando de acordo com o peso e a pelagem do animal. $\mathrm{O}$ procedimento padrão da empresa era: cordeiros e borregos, $350 \mathrm{~V}$ e $1 \mathrm{~A}$; em animais adultos e lanados (não era retirado $\mathrm{o}$ excesso de lã), o máximo utilizado era $750 \mathrm{~V}$ e $2 \mathrm{~A}$, por, no máximo, três segundos. Neste grupo foi avaliada a eficiência da insensibilização, da seguinte forma: imediatamente ao choque, o animal era colocado sobre a grade metálica e, então, avaliava-se o reflexo de endireitamento, a respiração rítmica (narinas e flanco) e o reflexo corneal. Após essa etapa, o animal era mantido em decúbito lateral para se efetuar a degola e a sangria por três minutos. As facas utilizadas eram trazidas pelo Zabeh e não eram afiadas durante o abate.

Foram colhidas, de cada animal, amostras de $6 \mathrm{~mL}$ de sangue imediatamente após a sangria, em tubos esterilizados sem anticoagulante, para obtenção do soro; mais $2 \mathrm{~mL}$ em outro tubo com anticoagulante etilenodiamino tetra-acetato de potássio (EDTA-K), para realização do eritrograma e da contagem total de leucócitos; e mais $2 \mathrm{~mL}$ em um frasco contento fluoreto para dosagem de glicose e lactato. Todas as amostras foram armazenadas e refrigeradas até serem centrifugadas a $2.200 \mathrm{rpm}$ durante 15 minutos. $\mathrm{O}$ soro e o plasma foram separados em três alíquotas em microtubos de plástico, identificados e mantidos a $-20^{\circ} \mathrm{C}$ até a realização das análises. Foram realizados os exames lactato desidrogenase (LDH), lactato, aspartato aminotransferase (AST), creatina quinase (CK), glicose, por espectofotometria seguindo as recomendações do fabricante $\left(\right.$ Labtest $^{\circledR}$ ), e cortisol, pela técnica de quimioluminescência, conforme Turpeinem e Hämäläinen (2013).

A eficiência da sangria foi realizada em ambos os grupos. O sangue foi coletado em um recipiente plástico com capacidade de cinco litros, durante três minutos cronometrados. Em seguida, o sangue foi pesado com identificação de cada animal, em uma balança eletrônica, e calculou-se a eficiência da sangria da seguinte forma:

eficiência da sangria $(\%)=$ volume de sangue $(\mathrm{kg}) /$ peso vivo animal $(\mathrm{kg}) \times 100$. 
Os dados foram submetidos à análise de variância, seguidos de teste de comparação de médias pelo teste de Tukey 5\%, pelo programa estatístico Sisvar.

\section{RESULTADOSE DISCUSSÃO}

O tempo de 20 segundos após a degola foi estabelecido conforme proposto por Gregory e Wotton (1984), que observaram morte cerebral em 14 segundos após a degola, e pela impossibilidade de acompanhar por um tempo maior, por se tratar de um abate comercial. Nenhum dos animais, no grupo CI, apresentou sinais de sensibilidade após a eletronarcose. Já no grupo SI, 20 segundos após a degola, verificaramse quedos 51 animais, $22(43 \%)$ apresentaram reflexo de endireitamento, e 11 (23\%) de endireitamento e vocalização, o que sugere que $66 \%$ dos animais estavam conscientes.

Rodriguez et al. (2012) estudaram a perda da consciência em cordeiros abatidos sem insensibilização por meio do monitorIoC-view ${ }^{\circledR}$, utilizado para avaliar a profundidade da anestesia em pacientes humanos, e concluíram que os cordeiros perdiam a consciência entre 22 e 82 segundos após a degola e que essa diferença poderia ser atribuída à eficiência da sangria.

Sabow et al. (2016) relataram que cabritos abatidos sem insensibilização perdem o reflexo pupilar somente 2,44 minutos após a degola; o mesmo tempo, segundo Khalid et al. (2015), necessário para a completa exsanguinação (Khalid et al., 2015). E é justamente este fato que tem gerado polêmicas na discussão sobre o abate halal sem insensibilização, a questão sobre o bemestar animal, ou seja, o tempo em que o animal permanece consciente em sofrimento. Outro ponto importante que deve ser considerado é a dor do corte no momento do abate nos animais sem insensibilização. Infelizmente ainda não há um método padrão para avaliar a dor no momento do abate (Aghwan et al., 2016).

Os consumidores de carne halal, apesar de preferirem que o abate seja realizado sem insensibilização, aceitam a insensibilização desde que o método de atordoamento não resulte em morte, lesões físicas, ou interfira na eficiência da sangria (Fuseini et al., 2017), porque acreditam que a insensibilização possa reduzir a quantidade de sangue expelida durante a sangria (Nakyinsige et al., 2013). Neste trabalho, avaliou-se a eficiência da sangria com os animais posicionados horizontalmente por um período de três minutos, pois, segundo Khalid et al. (2015), a exsanguinação é completa em dois minutos. Verificou-se que o grupo SI apresentou perda de sangue de $1,82 \mathrm{~kg}$, enquanto o grupo CI de $1,59 \mathrm{~kg}$ $(\mathrm{P}<0,01)$, resultado semelhante ao encontrado por Kiran et al. (2019).

$\mathrm{Na}$ eficiência da sangria também se observou diferença estatística significativa $(\mathrm{P}<0,01)$, na qual o grupo CI apresentou eficiência de $3,75 \%$ e o grupo SI, de $4,30 \%$, diferentemente dos resultados encontrados por Kiran et al. (2019), quando compararam o abate em cordeiros com e sem insensibilização elétrica. Warris (1984) estabeleceu que a perda de sangue durante a sangria em ovinos é, em média, $4 \%$ do seu peso vivo e que a menor eficiência da sangria nos animais que são insensibilizados se deve à parada cardíaca.

Outro fato importante relatado por Khalid et al. (2015) é com relação ao posicionamento do animal durante a sangria, pois, quando é pendurado, há um aumento da perda de sangue, em média, de $0,4 \mathrm{~kg}$. Os autores observaram que, no grupo sem insensibilização, a perda de sangue é mais lenta nos primeiros 10 segundos, porém não se encontrou diferença estatística significativa entre os grupos, com eficiência da sangria de $3,65 \%$ no grupo sem insensibilização e de 3,58\% com insensibilização. Acredita-se que os resultados divergentes encontrados em diversos trabalhos sejam devido a fatores como presença de conteúdo ruminal (Khalid et al., 2015), sangue aderido à lã e posicionamento do animal durante a sangria (Velarde et al., 2014).

Nenhum dos parâmetros hematimétricos excedeu os valores fisiológicos para a espécie, porém houve diferença estatística significativa para hemácias, hemoglobina e contagem total de leucócitos, como demonstra a Tab. 1. 
Tabela 1. Valores do eritrograma e contagem de leucócitos totais de ovinos abatidos pelo método halal com insensibilização elétrica (CI) e sem insensibilização (SI)

\begin{tabular}{lcccc}
\multicolumn{1}{c}{ Parâmetros } & Valor de referência & Grupo CI & Grupo SI & Valor de P \\
\hline Hematócrito $(\%)$ & $27-45$ & 33,35 & 32,23 & $0,10^{\mathrm{ns}}$ \\
Hemoglobina $(\mathrm{g} / \mathrm{dL})$ & $9-15$ & 14,02 & 13,25 & $0,05^{*}$ \\
Hemácias x $10^{6} / \mu \mathrm{L}$ & $9-15$ & 12,63 & 11,65 & $0,01^{* *}$ \\
Leucócitos $(/ \mu \mathrm{L})$ & $4.000-12.000$ & 4187,43 & 5987,84 & $0,01^{* *}$ \\
\hline
\end{tabular}

${ }^{\mathrm{ns}}$ Não significativo. * Significativo 5\%.** Significativo 1\%. Valor de referência: Schalm (2008).

A diferença estatística significativa no número de hemácias e na concentração de hemoglobina no grupo CI pode ser devido ao aumento da pressão arterial provocada pelo choque elétrico, que, segundo Kirton et al. (1978), aumenta em até 3,5 vezes, em um tempo médio de 11 segundos após o atordoamento. Além disso, na insensibilização elétrica, ocorre parada respiratória pela tetanização diafragmática, o que pode levar à contração esplênica, como mecanismo compensatório.

Já com relação ao número total de leucócitos, observou-se diferença estatística significativa entre os grupos, provavelmente devido ao maior esforço físico e ao estresse durante a contenção no grupo SI, pois sabe-se que a adrenalina liberada durante o estresse ou o exercício intenso faz com que ocorra a desmarginalização de leucócitos da parede do vaso para a corrente sanguínea, aumentando a contagem total dessas células (Thrall, 2007). Os biomarcadores séricos têm sido bastante utilizados, por fornecerem informações sobre as alterações fisiológicas que ocorrem devido ao estresse provocado pelo abate. Os resultados dessa análise estão apresentados na Tab. 2.

Tabela 2. Valores médios das análises bioquímicas séricas em ovinos abatidos pelo método halal com insensibilização elétrica (CI) e sem insensibilização (SI)

\begin{tabular}{lcccc}
\hline \multicolumn{1}{c}{ Parâmetros } & Valor de referência & Grupo CI & Grupo SI & Valor de P \\
\hline AST (UI/L) & $60-280$ & 137,31 & 141,00 & $0,77^{\text {ns }}$ \\
Creatina quinase - CK (U/L) & $8,1-12,9$ & 436,74 & 575,03 & $0,01^{* *}$ \\
Lactato desidrogenase - LDH (U/L) & $238-440$ & 1185,58 & 1174,58 & $0,86^{\text {ns }}$ \\
Glicose $(\mathrm{mg} / \mathrm{dL})$ & $50-80$ & 58,49 & 68,31 & $0,08^{\text {ns }}$ \\
Cortisol $(\mu \mathrm{g} / \mathrm{dL})$ & $1,88-2,60$ & 5,99 & 6,30 & $0,66^{\mathrm{ns}}$ \\
Lactato $(\mathrm{mmol} / \mathrm{L})$ & $1-1,33$ & 1,96 & 3,87 & $0,01^{* *}$ \\
\hline
\end{tabular}

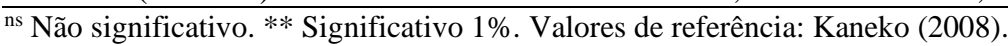

As enzimas AST, lactato desidrogenase (LDH) e creatina quinase (CK) são utilizadas para avaliarem fadiga e dano muscular (Russel e Russel, 2007). Não foram observadas alterações séricas para AST e LDH. Houve diferença estatística significativa para $\mathrm{CK}$, com valores aumentados para ambos os grupos. Conforme Nakyinng et al. (2014), as enzimas CK e LDH sugerem estresse, fadiga ou lesão muscular (estresse físico), que, associados ao aumento sérico do cortisol, em ambos os grupos, indicam que houve estresse no momento do abate nos animais com e sem insensibilização. Provavelmente o manejo de contenção em ambos os grupos, principalmente no grupo sem insensibilização, tenha acarretado estresse, o que levou ao aumento dessas enzimas.
Com relação ao lactato, observou-se diferença significativa entre os grupos, provavelmente como resultado de uma rápida glicólise anaeróbica (Grandin, 1998). Quanto à concentração sérica de glicose, observaram-se valores basais para ambos os grupos, sem diferença estatística significativa. Já Sabow et al. (2016) verificaram diferença estatística significativa, na dosagem sérica de glicose, em cabritos abatidos com e sem insensibilização, com valores mais altos para o grupo sem insensibilização. Em um outro trabalho, realizado por Kiran et al. (2019), verificou-se diferença estatística significativa, com maior valor de glicose para cordeiros que foram insensibilizados quando comparados aos não insensibilizados. 
Essas diferenças revelam que a utilização da glicose como biomarcador de estresse deve ser realizada com cautela, pois sofre influência de variáveis como jejum mais prolongado (Stewart et al., 2018), variação metabólica individual (Choe e Kim, 2014), bem como variações durante a resposta ao estresse. Segundo Seyle (1956), o estresse desenvolve uma resposta do organismo denominada por ele de Síndrome de Adaptação Geral (SGA), a qual é dividida em fases: na primeira fase, denominada de Fase de Alarme, ocorre aumento do cortisol e diminuição da glicose; na segunda fase, denominada de Fase de Resistência, os valores de cortisol e glicose se mantêm acima dos valores basais, até se entrar na Fase de Exaustão. Portanto, devido a uma gama de variáveis, a glicose sérica não pode ser utilizada individualmente na indicação do estresse.

\section{CONCLUSÃO}

Os animais sem insensibilização apresentaram sinais de endireitamento e vocalização após 20 segundos da degola. Os biomarcadores séricos LDH, CK, lactato e cortisol indicaram que houve estresse no abate halal com e sem insensibilização. A eficiência da sangria é maior em animais sem insensibilização.

\section{REFERÊNCIAS}

AGHWAN, Z.A.; BELLO, A.U.; ABUBAKAR, A.A. et al. Efficient halal bleeding, animal handling, and welfare: a holistic approach for meat quality. Meat Sci., v.121, p.420-428, 2016.

BRASIL. Ministério da Agricultura, Pecuária e Abastecimento. Instrução Normativa n. 3, de 17 de janeiro de 2000. Aprova o Regulamento Técnico de Métodos de Insensibilização para o abate Humanitário de animais de Açougue. Lex. Diário Oficial da União. Brasília, 24 de janeiro de 2000. Seção I, p.14-16.

CHOE, J.H.; KIM, B.C. Association of blood glucose, blood lactate, sérum cortisol levels, muscle metabolites, muscle fiber type composition, and pork quality traits. Meat Sci., v.2, p.137-142, 2014.

FAROUK, M. M.; AL-MAZEEDI, H.M.; SABOW., A.B. et al. Halal and Kosher slaughter methods and meat quality: A review. Meat Sci., v. 98, n.3, p. 505-519, 2014.
FUSEINI, A.; KNOWLES, T.G.; LINES, J.A. et $a l$. The stunning and slaughter of cattle within the EU: a review of the current situation with regard to the Halal market. Anim. Welfare, v.25, p.365376, 2016.

FUSEINI, A.; WOTTON, S.B.; HADLEY, P.J.; KNOWLES, T.G. The compatibility of modern slaughter techniques with halal slaughter: a review of the aspects of 'modern' slaughter methods that divide scholarly opinion within the Muslim Community. An. Welfare, v.26, p.301310, 2017.

GRANDIN, T. On-farm conditions that compromise animal welfare that can be monitored at the slaughter plant. Meat Sci., v.132, p.52-58, 2017.

GRANDIN, T. The feasibility of using vocalization scoring as na indicador of poor welfare during slaughter. Appl. Anim. Behav. Sci. J., v.56, p.121-128, 1998.

GREGORY, N.G.; FIELDING, H.R.; VONWENZLAWOWICZ, M.; HOLLEBEN, K. Time to collapse following slaughter without stunning in cattle. Meat Sci., v.85, p.66-69, 2010.

GREGORY, N.G.; WOTTON, S.B. Sheep slaughtering procedures 2: time to loss of brain responsiveness after exsanguinations or cardiac arrest. Br. Vet. J., v.140, p.354-360, 1984.

KANEKO, J.J. Clinical biochemistry of domestic animals. 6.ed. San Diego: Academic Press, 2008.

KHALID, R.; KNOWLES, T.J.; WOTTON, S.B. A comparison of blood loss during the halal slaughter of lambs following traditional religious slaughter without stunning, electric head-only stunning and post-cut electric head-only stunning. Meat Sci., v.110, p.15-23, 2015.

KIRAN, M.A.; NAVEENA, B.M.B.; SMRUTIREKHA, M. et al. Traditional halal slaughter without stunning versus slaughter with electrical stunning of sheep (Ovis aries). Meat Sci., v.148, p.127-136, 2019.

KIRTON, A.H.; BISHOP, W.H.; MULLORD, M.M.; FRAZERHURST, L.F. Relationships between time of stunning and time of throat cutting and their effect on blood pressure and blood splash in lambs. Meat Sci., v.2, p.199-206, 1978. 
NAKYINSIGE, K.; CHE MAN, Y.B.; AGHWAN, Z.A. et al. Review: stunning and animal welfare from Islamic and scientific perspectives. Meat Sci., v.95, p.352-361, 2013.

NAKYINSIGE, K.; SAZILI, A.; ZULKIFLI, I. et al. Influence of gas stunning and halal slaughter (no stunning) on rabbits welfare indicators and meat quality. Meat Sci., v.98, p.701-708, 2014.

RELATÓRIO detalhado sobre as exportações de carne brasileira. São Paulo: ABIEC, 2017. Disponível em:

http://www.abiec.com.br/estatísticas.asp.

Acessado em: 7 jan. 2018.

RODRIGUEZ, P.; VELARDE, A.; DALMAU, A.; LLONCH, P. Assessment of unconsciousness during slaughter without stunning in lambs. An. Welfare, v.21, p.75-80, 2012.

RUSSELL, K.E.; ROUSSEL, A.J. Evaluation of the ruminant serum chemistry profile. Vet. Clin. N. Am. Food Pract., v.3, p.403-426, 2007.

SABOW, A.B.; GOH, Y.M.; ZULKIFLI, I. et al. Blood parameters and electroencephalographic responses of goats to slaughter without stunning. Meat Sci., v.121, p.148-155, 2016.
SEYLE, H. The stress of life. New York: McGraw-Hill, 1956. 324p.

STEWART, S.M.; MCGILCHRIST, P.; GADNER, G.E.; PETHICK, D.W. Lamb loin tenderness is not associated with plasma indicators of preslaughter stress. Meat Sci., v.137, p.147-152, 2018.

THRALL, M.A. Hematologia e bioquímica clínica veterinária. São Paulo: Roca, 2007.

VELARDE, A.; RODRIGUEZ, P.; DALMAU. A. et al. Religious slaughter: evaluation of current practices in selected countries. Meat Sci., v.96, p.378-287, 2014.

WARRISS, P.D. Exsanguination of animals at slaughter and the residual blood content of meat. Vet. Rec., v.115, p.292-295, 1984.

WORLD Organization for Animal Health. Animal Welfare, chapter slaughter of animals. In: TERRESTRIAL ANIMAL HEALTH CODE, 2019. Available in: https://www.oie.int/standardsetting/terrestrial-code/access-online. Acessed in: 9 Dec. 2019. 